Questions vives

\section{Questions Vives}

Recherches en éducation

$\mathrm{N}^{\circ} 33 \mid 2020$

Visées heuristiques, praxéologiques et critiques dans les recherches participatives en éducation

\title{
Aliénation et émancipation en recherche, détour par la santé mentale et la recherche " avec »
}

Gilles Monceau, Marguerite Soulière et Cinira Magali Fortuna

\section{(2) OpenEdition}

\section{Journals}

Édition électronique

URL : http://journals.openedition.org/questionsvives/4646

DOI : $10.4000 /$ questionsvives. 4646

ISSN : 1775-433X

Éditeur

Université Aix-Marseille (AMU)

Édition imprimée

Date de publication : 15 décembre 2020

ISBN : 978-2-912643-57-5

ISSN : 1635-4079

\section{Référence électronique}

Gilles Monceau, Marguerite Soulière et Cinira Magali Fortuna, «Aliénation et émancipation en recherche, détour par la santé mentale et la recherche « avec » », Questions Vives [En ligne], N³3। 2020, mis en ligne le, consulté le 25 mars 2021. URL : http://journals.openedition.org/questionsvives/ 4646 ; DOI : https://doi.org/10.4000/questionsvives.4646

Ce document a été généré automatiquement le 25 mars 2021.

\section{(i) $९$}

Questions Vives est mis à disposition selon les termes de la licence Creative Commons Attribution Pas d'Utilisation Commerciale - Pas de Modification 4.0 International. 


\title{
Aliénation et émancipation en recherche, détour par la santé mentale et la recherche « avec »
}

\author{
Gilles Monceau, Marguerite Soulière et Cinira Magali Fortuna
}

\section{Introduction}

1 Intégrer une visée critique émancipatrice au travail de recherche, n'est-ce pas considérer implicitement que celui-ci est généralement aliéné ? Cette observation nous conduit par association à faire des parallèles entre le contexte institutionnel actuel de la pratique de recherche et celui qui cadrait les pratiques psychiatriques avant les transformations profondes qui ont eu cours depuis la deuxième moitié du vingtième siècle, en Europe, en Amérique du Nord et en Amérique du Sud. Cette association peut être jugée incongrue ou provocatrice, elle est cependant pertinente au regard d'une certaine histoire des sciences sociales.

2 Cet article est rédigé par une anthropologue canadienne, une spécialiste de santé collective brésilienne et un analyste institutionnel français travaillant en transversalité aux secteurs de la santé, l'éducation et le travail social. Il propose tout d'abord de revisiter le processus historique de réforme de la psychiatrie pour présenter ensuite une contribution aux réflexions et expérimentations en cours dans les sciences humaines et sociales visant à élaborer des démarches qui conjuguent visées praxéologique, heuristique et critique. Pour les auteurs, ce projet est passé concrètement par la construction d'un réseau scientifique international et pluridisciplinaire en capacité de mener une critique collective des cadres scientifiques institués. C'est donc aussi de la création et de la dynamique de ce réseau qu'il sera question. Ici, la transformation (et l'émancipation) des pratiques de recherche commence par l'interrogation critique des chercheurs sur leurs propres aliénations et leur recherche commune/collective de voies alternatives. 


\section{Aux origines d'une réflexion}

3 Si l'on accepte la définition selon laquelle l'aliénation se caractérise, pour un individu, par le fait d'être en situation d'impuissance et d'absence de sens et l'émancipation comme visant à échapper, par un travail de conscientisation et de réflexivité critique, aux déterminismes qui conditionnent la vie sociale (Marcel \& Broussal, 2017), les pratiques de recherche sont autant concernées que les pratiques psychiatriques et plus largement de santé mentale. C'est aussi ce que montre l'histoire des courants de recherche-action, porteuse de projets de transformation sociale conjuguant visées praxéologiques et heuristiques (Monceau, 2015).

4 Des sociologues, philosophes, psychiatres et psychanalystes (Goffman, 1961 ; Cooper, 1967 ; Basaglia, 1968 ; Foucault, 1972, 1975 ; Deleuze et Guattari, 1972) ont posé les bases d'une analyse critique de l'institutionnalisation et de la psychiatrisation des personnes en rattachant la maladie mentale à des maux sociaux induits par le capitalisme et l'Église. Cette littérature des années 1960-70 se prolonge dans les années 2000 par des analyses des enjeux politiques, économiques et sécuritaires du champ de la santé mentale (Demailly \& Autès, 2012 ; Labouret, 2012). La santé mentale, comme institution, n'est pas qu'une affaire sanitaire. Comme le montrent de nombreux travaux en sciences humaines et sociales, elle dit beaucoup sur l'organisation des sociétés humaines.

5 En Europe et en Amérique du Nord, après la seconde guerre mondiale, l'ordre médical et ses pratiques psychiatriques d'internement sont de plus en plus contestés et perdent leur monopole au profit de pratiques novatrices de professionnels (principalement psychiatres et infirmiers) qui, de l'intérieur proposent des manières alternatives de faire (Daumezon \& Koechlin,1952). Mais ces critiques internes et externes de l'institution psychiatrique débordent largement le cadre des établissements spécialisés, elles interrogent plus largement l'état de plus ou moins grande aliénation des individus dans les sociétés où ils vivent. La critique s'élargit au système politique, souvent dénoncé comme patriarcal et conservateur, et au système économique du capitalisme (Soulière, Gentelet \& Coman, 2014).

6 Ces ruptures dans la manière de penser le rapport entre individus et société se retrouvent dans le développement de la réflexivité critique en recherche par les chercheures féministes dans les années 1970 (Pillow, 2003), et ceux ayant le souci de la portée émancipatoire de leurs travaux (Denzin \& Lincoln, 2005 ; Mc Cabe \& Holmes, 2009). Il s'agissait pour eux de déconstruire les assises des pratiques reconnues scientifiques du fait de leur neutralité et de leur objectivité. Les experts produisant des discours de vérité à distance des réalités vécues et, surtout, du point de vue de l'homme blanc de classe moyenne supérieure, n'ont plus été les seuls dans le champ de la production des connaissances. D'autres points de vue ont produit d'autres analyses et ouvert à des prises de conscience concernant des processus d'aliénation jusque-là non perçus comme tels.

7 Ces ouvertures dans le champ de la recherche d'une part et de la santé mentale d'autre part coïncident dans leurs visées de dés-enfermement social et politique. Ce vaste mouvement de dés-institutionalisation apparait comme une libération, un affranchissement de personnes socialement réduites à leurs écarts à la normalité médicale et légale. La mise à l'écart physique institutionnalisée de la société, en particulier en hôpital psychiatrique, fut certes au cœur d'un dispositif (Foucault, 1975), mais aussi l'inscription des personnes dans des cases diagnostiques, des définitions 
comportementales, des capacités mentales, toutes assorties de règles de conduites acceptables dont dépendent les possibilités d'inclusion dans le monde "sain » et « productif » de la société (Hacking, 2002).

8 L'Organisation Mondiale de la Santé propose, dans sa Constitution de 1946, la définition suivante : "La santé est un état de complet bien-être physique, mental et social, et ne consiste pas seulement en une absence de maladie ou d'infirmité». Lors de sa rencontre d'Ottawa, en 1986 (qui donna le coup d'envoi à l'idée de promotion de la santé plutôt que de limiter les discours et les pratiques à la prévention des maladies), l'OMS mit un effort particulier à reconnaitre l'importance des actions de promotion de la santé mentale (Charte d'Ottawa, 1986).

Dans le plan d'action Psychiatrie et Santé mentale 2005-2008 du ministère français des solidarités et de la santé, la santé mentale est conçue en trois dimensions : «la santé mentale positive qui recouvre l'épanouissement personnel, la détresse psychologique réactionnelle qui correspond aux situations éprouvantes et aux difficultés existentielles, les troubles psychiatriques qui réfèrent à des affections diagnostiques renvoyant à des critères, à des actions thérapeutiques ciblées et qui correspondent à des troubles de durée variable et plus ou moins sévères et handicapants »-(p.5). Cette conception large de la santé mentale concerne donc l'ensemble de la population. De nombreux travaux portant sur les risques psychosociaux, l'usure professionnelle et la souffrance professionnelle ont d'ailleurs été menés dans différents milieux professionnels (Dejours, 2009). Ils pointent les effets négatifs des nouvelles formes de gestion sur la santé mentale des professionnels (de Gaulejac, 2005). Les chercheurs n'échappent pas à ces évolutions comme le montrent un nombre croissant de recherches au plan international (De Sousa, 2015 ; Drucker-Godard, Fouque, Gollety \& Le Flanchec, 2013). De fait, leur santé mentale est fragilisée par les effets de la concurrence scientifique mondiale.

\section{Transformer les pratiques institutionnelles pour désaliéner}

10 Le secteur de la santé mentale est pionnier dans la manière de considérer le patient et plus précisément dans la manière de construire des dispositifs thérapeutiques lui donnant une place de sujet plutôt que d'objet. Ainsi, la psychothérapie institutionnelle (Daumezon et Koechlin, France), la psychiatrie démocratique (Basaglia, Italie) et l'antipsychiatrie (Cooper, Grande Bretagne) ont contribué, à partir des années 1940 en Europe à transformer les hôpitaux psychiatriques traditionnels à partir d'une transformation des pratiques professionnelles elles-mêmes. Il s'agissait alors de soigner l'hôpital pour qu'il soit en mesure de soigner les patients.

11 Goffman avait montré dans son ouvrage Asiles (1961), dans lequel il a élaboré le concept d'institution totale, comment l'hôpital psychiatrique peut produire des suradaptations dans les comportements des internés, celles-ci les empêchant durablement de le quitter. Foucault, pour sa part, n'a cessé de rapprocher les fonctionnements de l'hôpital psychiatrique et de la prison en insistant sur leurs dimensions disciplinaires communes, disciplines appliquées aux esprits comme aux corps $(1972,1975)$. Bien que le malade ne cesse pas de résister, selon des modalités plus ou moins dissimulées, le traitement qui lui est appliqué fait de lui un objet. Les psychiatres Daumezon et 
Koechlin n'ont d'ailleurs pas hésité en 1952 à rapprocher dans leur analyse l'hôpital psychiatrique et le camp de concentration.

12 En donnant un nouveau statut au malade, en réformant les pratiques et les organisations, en tentant de désinstitutionnaliser les établissements comme les esprits, le secteur psychiatrique a sans doute été le domaine professionnel du «travail sur autrui » (Dubet, 2002) ou des « métiers de la relation» (Demailly, 2008) dans lequel des professionnels ont transformé le plus durablement leurs pratiques depuis l'intérieur de leurs institutions, ceci résolument en appui sur l'extérieur. Ainsi, les Centres d'Entraînement aux Méthodes de l'Éducation Actives, mouvement d'éducation populaire, ont formé les premiers infirmiers psychiatriques à partir de 1949 à mener, avec les malades, des activités rompant avec la routine psychiatrique (Le Henaff, 1949).

13 Au Brésil, l'histoire de la psychiatrie est liée à des influences européennes mais la désinstitutionalisation y a pris des formes singulières qui restent en grande tension. Le courant de la Santé collective y a largement contribué en renouvelant le paradigme du soin. Le Système Unique de Santé (SUS) et ses principes d'intégralité (prise en compte nécessaire de l'ensemble de la personne soignée) et d'humanisation (reconnaissance du patient comme un sujet détenteur de droits) reposent ainsi largement sur la démarche et les références de la Santé collective, dont le courant français d'analyse institutionnelle (Lourau, 1970).

Plus spécifiquement, le mouvement brésilien « anti-asilaire » ou " antimanicomial » qui lutte pour la fermeture des asiles psychiatriques apparaît dans les années 1980 dans la conjoncture de re-démocratisation après deux décennies de dictature. Ce mouvement, toujours en action aujourd'hui milite pour la libération de l'expérience de la folie par la transformation de » l'imaginaire social de la folie ». En prônant une société sans asiles, le mouvement promeut le passage « d'un rapport de domination, [...] d'enfermement et de mortification à un rapport de cohabitaiton entre les différences, de circulation dans le territoire, d'expression et de non-répression" (Brum Schaeppi, 2016, p. 6). L'intervention en santé mentale se voit ainsi transformée et décloisonnée avec l'inclusion des personnes utilisatrices dans les décisions portant sur l'organisation des soins qui les concernent. La reconnaissance de celles-ci comme des personnes «libres, égales et différentes ", comme des "sujets politiques " amène à une transformation de la société pour qu'elle se libère de la discrimination et se fasse inclusive, et des services en santé mentale pour qu'ils soient basés sur le respect des droits. Ce mouvement vise l'émancipation des personnes vivant avec une condition mentale différente et la prise en compte de l'expérience de leur souffrance (Brum Schaeppi, 2016).

Au Canada, comme en Europe, c'est à la suite de la deuxième guerre mondiale et avec la comparaison entre les asiles psychiatriques et les camps de concentration, que l'enfermement et l'état de reclus sont devenus intolérables (Castel, 1971, Dorvil, 2005). C'est par sa médicalisation, la maladie mentale est reconnue et mérite tous les égards de la médecine, que s'amorce d'abord paradoxalement son humanisation. À partir des années 1950, des médicaments psychotropes de plus en plus efficaces (on parle de contention chimique) permettent aux personnes souffrant de troubles mentaux de vivre dans la communauté. C'est ainsi que, dans les années 1960, se trouve facilité le grand dés-enfermement, le mouvement de la dés-institutionalisation, de la déshospitalisation.

On ferme les asiles, on diminue les lits dans les hôpitaux et, au Canada, on redirige les budgets dans des programmes communautaires (Mallette, 2003). Les traitements 
médicamenteux ne se suffisent en effet pas à eux-mêmes et nécessitent un travail de socialisation pour permettre aux personnes de retrouver une place dans la cité. Le traitement social et le traitement chimique de la maladie mentale ne s'opposent pas par définition bien que les débats restent souvent vifs.

\section{Transformer les pratiques de recherche pour émanciper}

17 Ces transformations majeures peuvent être lues comme des processus de désinstitutionnalisation (de la psychiatrie répressive) et d'institutionnalisation, ou réinstitutionnalisation (de la santé mentale) et leurs effets font l'objet de recherche sur deux horizons épistémologiques. Comme la production des connaissances est toujours située dans l'histoire d'un chercheur et d'une discipline et dans un contexte sociopolitique (Harraway, 1988), les objets de recherche sont façonnés dans des préoccupations et des particularités, dans des lieux et des époques donnés. Lorsque les chercheurs s'intéressent au regroupement des ex-patients psychiatrisés, le Mad (Liberation) Movement (1972) ou un peu plus tard le Mad Pride (1993), deux tendances se dessinent.

D'une part, des points de vue de la psychiatrie, de la psychologie et d'un courant du travail social, les effets bénéfiques des groupes qui s'en réclament concernent surtout les pratiques thérapeutiques alternatives, particulièrement celles développées autour du concept de pairs aidants. Ici, c'est entre individus partageant un passé et un vécu similaires de maladie mentale qu'un processus d'autonomisation, de prise en charge individuelle de sa propre destinée va s'amorcer et potentiellement se réaliser (Emerick, 1996). Les groupes rattachés au Mad Movement sont reconnus par ces disciplines dans ce qu'ils rejoignent les visées de bien-être individuel (et de diminution de coûts dans le contexte récurrent de coupures des budgets de santé au Canada) qui sont propres à celles-ci.

D'autre part, des points de vue de disciplines qui s'intéressent d'entrée de jeu aux dimensions collectives des phénomènes (sociologie, sciences politiques, et une vision structurelle du travail social), le Mad Movement est avant tout un mouvement social qui travaille au corps une société, ses représentations, ses politiques, ses lois, ses programmes scolaires. Ce mouvement ancre sa raison d'être dans une double mission. D'abord, éduquer, conscientiser la majorité de «normaux» de l'oppression, la discrimination et la souffrance vécues par les personnes affectées de détresse psychologique ou d'affections psychiatriques; et celle de faire reconnaitre les troubles mentaux autrement que comme une maladie (et un stigma) mais comme une condition mentale autre et possible dans le spectre de la diversité psychique.

C'est dans cette foulée que virent le jour les Mad Studies au Canada, qui posèrent sur cet horizon radical la recherche en santé mentale. Ce champ se définit comme « un projet de recherche, de production de connaissances et d'action politique dédiée à la critique et la transcendance des manières psychologisantes de penser, d'agir, d'interagir et d'être » (Le François, Menzies \& Rhéaume, 2013, p. 270) [traduction libre]. Aujourd'hui se pose la question des effets controversés et contradictoires de la production des connaissances dans les Mad Studies. Par exemple, les concepts majeurs de rétablissement et de pairs aidants, tous deux développés par des survivants de la psychiatrie ont servi 
d'appui à des politiques de retour à l'emploi, de coupures de subsides et de services professionnels (Beresford \& Russo, 2016). Ce n'était, bien sûr, pas l'objectif de ces militants.

21 En Europe, en Amérique du Nord et du Sud, les dispositifs de la santé mentale sont désormais variés et en partie démédicalisés, au sens où les médecins sont absents de nombreux dispositifs. En n'étant plus le monopole de la médecine, la santé mentale se trouve traversée de façon plus visible par différentes institutions (famille, religion, assistance sociale, entreprise privée, médias) et est objet de tensions entre différents groupes d'intérêt et entre différentes visions politiques dont certaines, sécuritaires, continuent à promouvoir l'enfermement des «fous» pour protéger la société. La tendance dominante est cependant à la participation citoyenne des personnes ciblées par les dispositifs de la santé mentale, ce qui se traduit par différentes initiatives (Gagné, 2009).

En faisant de nouveau l'association avec la recherche en sciences humaines et sociales, la perspective critiques doit aussi se traduire dans les dispositifs concrets par lesquels se fait la recherche si elle vise des transformations émancipatrices. Ceci suppose simultanément que les chercheurs développent une réflexivité critique sur leurs propres pratiques et implications (Monceau, 2013) et que les sujets de la recherche ne soient pas traités comme des objets dépourvus de capacités analytiques.

\section{Dans le domaine de la recherche en sciences humaines et sociales}

La démarche que nous souhaitons promouvoir est celle d'une recherche menée " avec » les sujets qu'elle concerne (Monceau, Soulière, 2016, 2017). En ce sens, les transformations advenues dans le champ de la santé mentale, tant dans les discours et les pratiques que les articulations de l'intervention et la recherche sont un point d'attache de notre réflexion/positionnement de chercheurs. La création du réseau de chercheurs international, interdisciplinaire et francophone Recherche avec est l'expression d'un sentiment d'urgence partagé par une diversité de chercheurs, ici et ailleurs, de contrer «l'enfermement » de la recherche en sciences humaines et sociales dans des disciplines académiques, des secteurs de recherche et des contextes sociopolitiques nationaux.

L'internationalisation de la recherche se fait par le développement de standards qui tendent à uniformiser les institutions et les pratiques scientifiques. Cela se concrétise à deux niveaux dans la vie professionnelle des chercheurs. Tout d'abord, les conditions de production des connaissances sont de plus en plus marquées par une compétition où l'excellence, notion fort discutable, devient la valeur d'échange. L'évaluation du travail $\mathrm{du}$ chercheur (qui détermine son accès aux financements) se fait en fonction de la quantité d'articles publiés après évaluation par les pairs, et la renommée des lieux de publications en fonction de leur facteur d'impact. Dans ce jeu de la division naturalisée entre excellents et non excellents, les revues anglophones sont les mieux cotées. Le métier de chercheur est progressivement imprégné par la crainte de la disqualification et du manque de fonds qu'induisent les conditions extérieures à celui-ci. La logique de la Nouvelle gestion publique, faite d'indicateurs de performance et de rationalisation 
des dépenses, transforme le sens même de l'activité scientifique. Les impératifs à court terme prennent le pas sur les travaux inscrits dans des temporalités longues.

La recherche est alors sommée de fournir des outils pour une pratique efficace et rentable. L'objectivation et la généralisation des résultats des recherches sont considérées comme garantes de leur scientificité et leur pertinence dans un contexte de coupures et de diminution des coûts (Bruno, 2015).

Dans cette conjoncture, un nombre grandissant de chercheurs souhaitent se regrouper, se soutenir, inventer ensemble et développer d'autres manières de faire la recherche qui vise une utilité sociale moins soumise aux injonctions productivistes. Il s'agit d'élaborer les moyens d'affranchir la recherche en sciences humaines et sociales, les chercheurs et les sujets de leur recherche de la colonisation d'un mode de pensée, souvent qualifié de "néolibéral ", au sens où la priorité serait donnée aux retombées économiques plutôt qu'à la démocratisation des sociétés et la qualité de la vie. Si la complexité du rapport entre savant et politique n'est pas une problématique nouvelle (Corcuff, 2011), il prend dans les années 2000 de nouvelles formes avec l'intensification de la pression financière et temporelle.

\section{Un exemple de mise en œuvre internationale}

Comme en santé mentale, le projet d'échapper à une aliénation faite d'impuissance face à des standards internationaux et à un productivisme qui ne fait pas sens pour les chercheurs appelle à imaginer des dispositifs appropriés. L'enjeu est de trouver la voie de l'émancipation en proposant d'autres modalités d'internationalisation orientées par la transversalité et la coopération.

Après une première rencontre d'une douzaine de chercheurs du Brésil, du Canada et de France dans le cadre du Congrès AREF 2013 de Montpellier (Monceau, 2017), le réseau Recherche avec a développé depuis 2013 des dispositifs qui outillent et nourrissent ses quelques 80 membres actifs, chercheurs, praticiens chercheurs, doctorants et intervenants du Brésil, du Canada, de France, du Portugal, d'Espagne et du Mexique. Véritable projet politique dans le contexte actuel, le réseau est une source de production savante collective mais aussi, et même en premier lieu, un espace d'expérimentation de collaborations créatives et solidaires.

L'élan premier qui donna le souffle aux textes d'appel pour le premier symposium organisé à l'Université d'Ottawa en 2014 fut naturellement le rassemblement, la mise en commun, le penser et le faire ensemble malgré les différences entre approches théoriques, domaines de recherche et pays d'origine. Le pari était que l'expérience commune de mener des recherches "avec " les personnes concernées par celles-ci constituerait un objet commun suffisant.

Un questionnaire a été diffusé auprès de participants potentiels qui débutait par ces mots: "Faire de la recherche avec: enjeux épistémologiques, méthodologiques, éthiques et pratiques en santé, éducation et intervention sociale ». Il fut demandé aux répondants de se prononcer sur le contenu même de cette première rencontre. Pour y arriver quatre questions assez simples leur étaient posées :

1. De quelle manière vous êtes-vous senti.e.s interpellé.e.s par l'appel ? Ou en quoi ce thème at-il suscité votre intérêt? 
2. Dans votre activité de recherche présente, passée ou future, que considérez-vous être de la recherche avec?

3. Auriez-vous deux textes ou auteurs à suggérer pour constituer la bibliographie du symposium?

4. Que souhaiteriez-vous aborder dans ce symposium?

31 Ce « avec » était intentionnellement ouvert et souple, permettant ainsi une diversité d'appropriations théoriques et méthodologiques. Cette polysémie du syntagme Recherche avec, posée dès le lancement du premier symposium, a tout de suite qualifié la nature des échanges : les visées n'étaient pas d'installer un nouveau cadre, mais bien de proposer un dispositif d'échanges et de partages d'expérience de recherches menées avec les personnes concernées par l'objet de la recherche.

L'étape suivante de l'organisation scientifique fut la compilation des réponses reçues d'une soixantaine de chercheurs brésiliens, canadiens, français et mexicains. Nous donnons ici quelques exemples de réponse :

33 A la question $1:$ "C'est une de mes pratiques habituelles de recherche. J'enseigne aussi un cours d'évaluation participative depuis deux ans. Mon intérêt est d'apprendre des pratiques des collègues, surtout au Canada, car ma pratique est surtout à l'international. Comprendre et partager les démarches pédagogiques employées pour l'enseignement. »

34 A la question 2: «Je ne crois pas qu'il existe une seule définition. Le «avec » se pose alors «avec qui ». Le qui pouvant être les personnes touchées par le problème mais aussi les utilisateurs potentiels des résultats. " La même réponse se prolonge en interrogeant aussi « quand » faire la recherche « avec ».

35 A la question 3: "Comment enseigner les approches participatives à l'Université? Comment survivre dans le milieu de la recherche compétitif en souhaitant réaliser des recherches participatives. Comment mobiliser les acteurs? Les enjeux de la recherche participative en Afrique. »

36 Ces réponses furent la base du lancement, sur une plateforme numérique, de huit groupes de discussions dans des espaces d'échanges virtuels sur une Plateforme interactive www.rechercheavec.com. Ces groupes répondaient à des thématiques qui avaient été dégagées de l'analyse des réponses au questionnaire : la place respective des différents acteurs, le praticien chercheur, les spécificités de l'écriture, la place de l'audio-visuel, la restitution, l'évaluation dans la recherche «avec» et finalement l'échange d'expériences et de pratiques. L'objectif était de créer des communautés de réflexion avant la rencontre à Ottawa pour le symposium. Ces échanges allaient assurer la préparation collective de huit ateliers thématiques lors desquels les chercheurs, praticiens chercheurs, doctorants et intervenants allaient partager entre eux les différents obstacles, les trouvailles, les succès et les défis rencontrés dans leur pratique de recherche.

37 Les ateliers furent ainsi préparés mais ils furent aussi transformés et pour certains détournés de leur parcours initialement prévu par des propositions faites et consenties sur place par les participants. Différemment des colloques aux présentations individuelles de résultats, le symposium qui a donné le coup d'envoi au réseau fut une véritable rencontre-échange autour des enjeux concrètement vécus dans les pratiques de la recherche dans nos différents pays. C'était une première occasion de réfléchir ensemble, de construire ensemble une idée, une démarche possible, un projet. 

2018) ont été des moments d'expérimentation d'autres manières d'organiser des rencontres entre chercheurs. Il s'y est inventé et vécu des liens inspirés par la coopération et la solidarité. Des chercheurs des pays d'accueil emmènent, par exemple, leurs collègues venant de pays étrangers sur leurs terrains pour rencontrer les personnes avec lesquelles des recherches sont menées. Les rencontres du réseau dans les différents pays adoptent donc des modalités spécifiques aux contextes et aux interlocuteurs locaux. Une prochaine semaine scientifique devait se tenir en 2020 au Mexique. La pandémie de COVID 19 nous a obligés à la reporter en 2021 d'une part et à imaginer de nouvelles modalités pour les échanges. Les différents ateliers s'organiseront en mode hybride (distance/présence). Le comité scientifique propose également des espaces d'échange et de soutien aux chercheurs et doctorants en lien avec ce même contexte. C'est aussi par cette souplesse d'adaptation aux événements et contextes d'une part et aux nécessités de ses membres d'autre part que la dynamique du réseau lutte contre l'isolement des chercheurs et les processus d'aliénation.

Une fois ces événements terminés, durant les deux années séparant deux rencontres, une pluralité de collaborations pour des activités savantes sont mises en œuvre dans et entre les différents pays: des compte rendu collectifs des ateliers, des ouvrages collectifs, des articles, des productions collectives de matériel pédagogique, de projets de recherche, etc...

\section{Dans les dispositifs de recherche}

Le réseau scientifique Recherche avec constitue donc un dispositif de travail dont la première qualité est de mettre en œuvre une transversalité entre disciplines universitaires à l'échelle internationale. La réflexivité critique guide l'organisation de ses rencontres bisannuelles dont des ateliers intersectoriels (éducation, santé et travail social) et internationaux sont les composants de base. Le terme "critique » est ici utilisé au sens d'une analyse des contextes sociaux, économiques, politiques et scientifiques influençant la pratique de recherche.

41 C'est dans le cadre du réseau qu'a été pensée une recherche portant sur les effets des pratiques et des discours dominants entourant la naissance sur le devenir parent et la parentalité au Brésil, au Canada et en France ${ }^{1}$. Il s'agissait de penser la naissance en dehors des cadres médicaux ordinaires, de la prédominance des logiques de risque et de diagnostic, des discours experts de prévention par la promotion de certaines représentations du bébé parfait voire de la mère parfaite. Le projet était ainsi de redonner sens à l'expérience et émanciper les parents et les intervenants par rapport à la centralité des indicateurs et facteurs de risques.

42 Les trois porteurs principaux de ce projet étant inscrits dans des disciplines universitaires différentes (santé collective, anthropologie et sciences de l'éducation et de la formation) et menant principalement leurs travaux dans des champs professionnels différents (santé, travail social et éducation), leur collaboration a été aussi la confrontation de points de vue différents dès l'écriture du projet pour la première demande de subventions. Ainsi le croisement des sensibilités théoriques et expérientielles (Paillé \& Mucchielli, 2010) des chercheurs a mené à poser les bases d'une recherche sur la naissance qui permettrait d'élargir les connaissances essentiellement médicales entourant l'arrivée d'un enfant en vue de mettre en lumière 
l'impact des composantes individuelles, institutionnelles, culturelles, sociales et politiques et l'importance d'en tenir compte dans l'intervention.

La mise en œuvre méthodologique fondée sur la volonté commune de construire des groupes de professionnels et de parents a posé la question des critères qui permettraient de rencontrer et documenter la diversité des approches de la naissance dans les différents milieux d'intervention et la diversité sociale et culturelle telles qu'elles s'actualisent dans chacun des pays. Fallait-il constituer ces groupes à partir de critères identiques dans chaque pays, par exemple caractériser les professionnels en fonction des structures sanitaires et éducatives les unes traditionnelles et les autres alternatives? Fallait-il prioriser dans les trois pays les différences socio-économiques des parents? Fallait-il organiser les rencontres pendant le temps de travail des professionnels ou bien en dehors? Fallait-il travailler avec des groupes de volontaires répondant à notre proposition ou bien chercher activement à y intégrer des professions (les médecins) ou des sexes (hommes, trans, etc.) peu représentés? Ces questions ne se posaient pas de la même manière dans les trois pays, c'est surtout les contraintes (culturelles, institutionnelles, économiques...) locales qu'il a fallu prendre en compte pour y répondre d'une manière à la fois harmonisée et différente selon les lieux.

Ces aléas lors de la construction du dispositif de recherche ne sont pas tant des freins que des opportunités pour mettre en évidence à la fois des tendances transnationales et des spécificités locales. Ainsi, deux approches contrastées de la naissance sont présentes dans les trois pays, l'une fondée sur le calcul des risques pour assurer le développement optimal de l'enfant et éviter les complications à la naissance -où la hiérarchisation des savoirs et la multiplication des interventions médicales sont justifiées- et une autre fondée sur la reconnaissance du caractère naturel (physiologique) de la naissance où les savoirs sont co-construits et l'expérience corporelle, émotionnelle, psychologique de la mère, inscrite dans un contexte conjugal, familial, culturel et social est au cœur des attentions professionnelles. Bien qu'elles soient mises en œuvre dans des dispositifs différenciés dans les trois pays et y occupent des places différentes, elles y servent de points de référence lorsqu'il s'agit de naissance et d'accompagnement au devenir parent et à l'arrivée d'un enfant.

Les échanges sur les vécus des participants parents et professionnels (ces derniers s'exprimant aussi souvent comme parents) ont été facilités par leur engagement dans un projet commun, celui de construire un questionnaire pouvant être renseignés par des parents des trois pays et ayant comme objectif de comprendre l'expérience de naissance et de devenir parent. Cette mobilisation collective sur une tâche commune a permis à tous d'expérimenter un décentrement par la confrontation à d'autres points de vue dans le groupe mais aussi à ce qui était rapporté par les chercheurs des idées exprimées dans les autres pays. Au fil des rencontres, des normes sociales (désir d'être ou de faire comme les autres), des malaises (sentiment que son corps a été traité comme une chose par exemple), des étonnements (à propos de réalités autres jusque-là insoupçonnées), des résistances (choix d'accoucher à domicile après des accouchements en milieu hospitalier par exemple) se sont exprimés. La naissance devient alors pour tous un objet complexe qui déborde largement son expérience personnelle et vient l'interroger en profondeur.

46 Ce travail de mise en commun des réalités décrites et expliquées dans les groupes nous a en effet permis de saisir l'impact des différents contextes sociopolitiques dans les interprétations subjectives des accompagnements et des interventions entourant la 
naissance. À titre d'exemple, si la césarienne est encore au Brésil une pratique ordinaire qu'une majorité de médecins présente comme la plus sûre à leurs patientes, elle est au contraire au Canada, en France et dans nombre de pays la marque d'une naissance « à risque » d'une part et de l'autre, une pratique à réguler. Les tentatives de diminution du taux de césarienne par les autorités sanitaires brésiliennes durant la période de la recherche, sont souvent vécues par les Brésiliennes moins fortunées comme une inégalité sociale les privant des meilleures conditions d'accouchement.

Il est ainsi apparu clairement que les différences sont aussi subjectives, le sentiment de sécurité éprouvé ou non par les femmes n'a par exemple rien d'universel ni de standardisé (Santana da Silva, 2019). Si la notion de risque est au cœur de la conception de la naissance dans les sociétés euro-américaines, les interprétations d'une naissance sécuritaire sont différenciées dans les trois pays concernés par l'enquête et selon les situations et points de vue variés des parents et des professionnels. Les dispositifs d'accompagnement et d'intervention qui en découlent diffèrent également. Cependant, dans les trois pays, lorsque l'accouchement se fait à l'hôpital, c'est une surveillance étroite de la normalité de son déroulement qui domine et conduit souvent à des interventions médicalisées (anesthésie péridurale, épisiotomie voire césarienne). Pour cette raison, des parents et des intervenants vont opter pour un autre lieu de naissance, où la place du père, l'autonomie de la mère et du couple, la relation thérapeutique sont garantes de sécurité quand l'accouchement est considéré à bas risque. Cette option n'est pas accessible aux mêmes conditions au Canada, en France et au Brésil et est encore le plus souvent le choix des milieux privilégiés ou alternatifs. Les mères immigrantes qui composaient majoritairement un groupe au Canada et un groupe en France ont, pour leur part, toutes suivi la filière hospitalière normalisée en invoquant la dimension sécuritaire que l'hôpital leur semble garantir.

On observe par ailleurs que les récits des professionnelles ${ }^{2}$ présentes auprès des mères en salle d'accouchement, montrent qu'elles cherchent à conjuguer dans leur pratique les dimensions humaines et sécuritaires, elles se heurtent alors à la fois aux revendications de certains parents et à l'autorité médicale (Bessaoud Alonso \& Monceau, 2019). Leur inconfortable posture d'entre deux, largement conditionnée par le milieu de pratique est apparue dans les groupes et lors d'une restitution des analyses avec les professionnelles françaises.

L'évocation de ces résultats de recherche illustre les différents niveaux d'émancipation que promet ce type de pratiques. D'abord, la confrontation des "normalités » nationales expose les chercheurs à la mise à l'épreuve des certitudes avec lesquelles ils problématisent leurs recherches. Les allants de soi ébranlés deviennent des brèches fertiles pour penser des réalités complexes, individuelles, sociales et culturelles à la fois locales et transnationales. Ensuite, ce travail collectif demande aux chercheurs de s'affranchir des normes temporelles de production de résultats et de demandes de financements qui évaluent leur qualité de chercheur. Le travail collectif et surtout les liens de confiance et d'amitié qui le dynamisent peuvent se lire comme une forme de transgression des normes et d'affranchissement institutionnel. Finalement, dans le cas précis de cette recherche s'intéressant à la naissance et à l'expérience vécue des parents et des professionnels, les résultats permettent de questionner la standardisation des pratiques et de reconnaître la validité, voire la dimension incontournable, de la prise en compte de l'expérience et du sens vécus par les sujets 
pour penser les interventions professionnelles (Soulière, Monceau, Fortuna, Mondain, Santana da Silva, Pilotti, 2020).

\section{Conclusion}

Les travaux pratiques et théoriques en santé mentale montrent que des alternatives y ont été imaginées à partir d'analyses critiques à la fois radicales (comme la comparaison entre l'hôpital psychiatrique et le camp de concentration) et réalistes (par la mise en œuvre concrète de modalités de soin nouvelles). C'est aussi à partir de ce champ qu'ont été imaginées de nouvelles perspectives de recherche débordant largement le secteur psychiatrique pour interroger l'institutionnalisation des sciences humaines et sociales.

51 À la suite et en dialogue avec les différents courants de recherche collaborative, recherche coopérative, recherche action, recherche intervention..., nous proposons de penser les visées, les pratiques et les enjeux politiques et sociaux de ces démarches de recherche sans les disjoindre. C'est cet objectif qui guide le développement du réseau Recherche avec.

52 L'objectif n'est pas de créer un nouveau courant de recherche mais de proposer un questionnement transversal commun qui, en reconnaissant la place centrale des sujets concernés, réforme notre manière de penser et pratiquer la recherche dans une période où la standardisation des normes éthiques, des procédures de collecte de données et de mise en forme des résultats peuvent tendre à déshumaniser les sciences humaines et sociales tout en affichant un discours de protection de l'humain.

Penser et mener la recherche "avec» les sujets impliqués, c'est donc peut-être transférer dans la pratique de recherche les acquis du mouvement de réforme du secteur de la santé mentale. En suscitant une dynamique collective et réflexive des chercheurs sur leurs propres pratiques, il s'agit de dénaturaliser certaines certitudes disciplinaires ou nationales. Ainsi, nos échanges internationaux font apparaitre que les procédures éthiques qui tendent parfois à étouffer la réflexion éthique elle-même n'ont rien d'universelles (Colinet \& Passos, 2015). Ce qui est interdit dans un pays ou un champ scientifique peut être permis ailleurs. Il en va de même à propos des outils méthodologiques. Les confrontations interdisciplinaires et internationales obligent en effet les chercheurs à expliciter leurs choix face à des interlocuteurs pour qui ceux-ci ne vont pas de soi.

Les rencontres intellectuelles et humaines incitent également les participants à examiner plus finement leurs implications dans les enjeux politiques qui traversent leur pratique de recherche (Fortuna, Mesquita, Matumoto, \& Monceau, 2016). Dans des formes toujours renouvelées, nous cherchons la voie d'une émancipation par l'analyse de nos conditions de production scientifique. C'est aussi résister collectivement à l'hégémonie d'une définition de l'excellence scientifique qui aliène la pensée scientifique en promouvant la concurrence et la productivité aux dépens de la coopération et du débat. Cette résistance passe par l'expérimentation d'autres manières de faire. 


\section{BIBLIOGRAPHIE}

Basaglia, F. (ed.)(1968). L'istituzione negate. Einaudi : Turin.

Beresford, P. et Russo, J. (2016). Supporting the sustainability of Mad Studies and preventing its co-option. Disability \& Society. 31(2), 270-274.

Bessaoud Alonso, P. \& Monceau, G. (2019). Des professionnels qui doivent faire avec les contradictions institutionnelles des politiques publiques. Connexions, 112.

Brum Schaeppi, P. (2016). Militer avec la folie : le pli politique du mouvement antimanicomial à Rio de Janeiro. Thèse de doctorat, Montréal, UQÀM.

Bruno, I. (2015). Défaire l'arbitraire des faits. De l'art de gouverner (et de résister) par les « données probantes ». Revue Française de Socio-Économie. 2 (Hors-série), 213-227.

Castel, R. (1971). L'institution psychiatrique en question. Revue française de sociologie. 12, 57-92. Colinet S. et I.F. Passos (2015). Éthique procédurale, entre petits arrangements et transgression : comparaison Brésil-France. Fractal: Revista de Psicologia. 27(3), 256-263.

Cooper, D. (1967). Psychiatry and antipsychiatry. London, New York : Tavistock.

Corcuff, P. (2011). Le savant et le politique, Sociologies [En ligne], "La recherche en actes, Régimes d'explication en sociologie", (consulté le 21 mars 2020. URL : http://journals.openedition.org/ sociologies/3533

Daumezon, G. et Koechlin, P. (1952). La psychothérapie institutionnelle française contemporaine. Anais portugueses de psiquiatria, 4(4), 271-312

Dejours, D. (2009). Souffrance en France. La banalisation de l'injustice sociale. Points : Paris.

Deleuze, G. \& Guattari, F. (1972). L'anti-oedipe. Paris : Minuit.

Demailly, L. (2008). Politiques de la relation. Approche sociologique des métiers et activités professionnelles relationnelles. Villeneuve d'Ascq : Septentrion.

Demailly, L. \& Autès, M. (dir.). (2012). La politique de santé mentale en France. Acteurs, instruments, controverses. Paris : Seuil.

Denzin, N.K. et Lincoln Y. (2005). Sage Handbook of qualitative Health Research. Thousand Oaks : Sage Edition.

De Sousa, A. P. (2015). Productivisme et souffrance chez les enseignants chercheurs au Brésil. Pensée plurielle. 38, 45-68.

Dorvil, H. (2005). Nouveau plan d'action : quelques aspects médicaux, juridiques, sociologiques de la désinstitutionalisation. Cahiers de recherche sociologique, 41- 42, 209-235.

Drucker-Godard, C., Fouque, T.,Gollety, M., Le Flanchec, A. (2013). Le ressenti des enseignantschercheurs : un conflit de valeurs. Gestion et management, 1 (4), 4-22.

Dubet, F. (2002). Le déclin de l'institution. Paris: Seuil.

Emerick, E. Robert (1996). Mad Liberation: The Sociology of Knowledge and the Ultimate Civil Rights Movement. The Journal of Mind and Behavior, Spring, 17(2), 135-160. 
Fortuna, C. M.; Mesquita, L.P.; Matumoto, S. \& Monceau, G. (2016). A análise de implicação de pesquisadores em uma pesquisa-intervenção na Rede Cegonha : ferramenta da análise institucional. Cadernos de Saúde Pública, 32(9) (en ligne).

Foucault, M. (1972). Histoire de la folie à l'âge classique. Paris : Gallimard

Foucault, M. (1975). Surveiller et punir, naissance de la prison. Paris, Gallimard

Gagné, J. (2009). La participation citoyenne en santé mentale : une expérience locale au Québec. Santé, Société et Solidarité, 2, 119-123.

de Gaulejac, V. (2005). La Société malade de la gestion. Idéologie gestionnaire, pouvoir managérial et harcèlement social. Paris: Seuil.

Goffman, E. (1961). Asylums: Essays on the Condition of the Social Situation of Mental Patients and Other Inmates. New York: Doubleday Anchor

Hacking, I. (2002). Mad Travelers : Reflections on the Reality of Transient Mental Illnesses, Harvard: Harvard University Press.

Harraway, D. (1988). Situated Knowledges : The Science Question in Feminism and the Privilege of Partial Perspective. Feminist Studies, 14(3), 575-599.

Labouret, O. (2012). Le nouvel ordre psychiatrique : guerre économique et guerre psychologique. Toulouse : Érès.

Le François, B. A., Menzies, R. \& Reaume, G. (ed.) (2013). Mad matters: a critical reader in Canadian mad studies. Toronto : Canadian Scholars' Press Inc.

Le Henaff, G. (1949). Stage des infirmiers des hôpitaux psychiatriques. Château de la Charbonnière du 22 au 29 septembre 1949. Vers l'Education Nouvelle, 37, 28-31.

Lourau, R. (1970). L'analyse institutionnelle. Paris : Minuit.

Mc Cabe, J.L. \& Holmes, D. (2009). Reflexivity, Critical Qualitative Research and Emancipation: a Foucaldian Perpective. Journal of Advanced Nursing, 65(7). 1518-1526.

Mallette, L. (2003). La psychiatrie sous influence. Santé mentale au Québec, 28(1) , 298-319.

Marcel, J.-F. \& Broussal, D. (dir.). (2017). Émancipation et recherche en éducation. Conditions de la rencontre entre science et militance. Paris : Éditions du Croquant.

Ministère des solidarités et de la santé (2004). Psychiatrie et Santé mentale. Paris http://solidaritessante.gouv.fr/IMG/pdf/plan_2005-2008.pdf

Monceau, G. (2013). Institutionnalisation de la réflexivité et obstacles à l'analyse de l'implication. Dans Béziat, Jacques (dir). Analyse de pratiques et réflexivité. Regards sur la formation, la recherche et l'intervention socio-éducative (p.21-32). Paris : L'Harmattan.

Monceau, G. (2015). La recherche-action en France : histoire récente et usages actuels. Dans Les chercheurs ignorants, Les recherches-actions collaboratives. Une révolution silencieuse de la connaissance (p. 21-31). Rennes : Presses de l'Ecole des hautes études en santé publique.

Monceau, G. (2017). Enquêter ou intervenir ? Effets de la recherche socio-clinique. Nîmes: Champ social. Monceau, G. \& Soulière, M. (2016). The democratization of research and knowledge: the Recherche avec network [editorial]. Online Brazilian Journal of Nursing, 15(4) : 594-598 (consulté le 27 mars 2020) URL : http://www.objnursing.uff.br/index.php/nursing/article/view/5907/html 
Monceau, G. \& Soulière, M. (2017). Mener la recherche avec les sujets concernés : comment et pour quels résultats ? Éducation et socialisation [En ligne], 45 (consulté le 27 mars 2020). URL : http://journals.openedition.org/edso/2525

Organisation mondiale de la santé (OMS) (1986). Charte d'Ottawa http://www.euro.who.int/ _-_data/assets/pdf_file/0003/129675/Ottawa_Charter_F.pdf?ua=1\&ua=1

Paillé, P. et Mucchielli, A. (2010). L'analyse qualitative en sciences humaines et sociales (3e éd.). Paris, France: Armand Colin.

Pillow, Wanda S. (2003). « Confession, Catharsis, or Cure ? Rethinking the Uses of Reflexivity as Methodological Power in Qualitative Research », Qualitative Study in Education, 2 , 175-196

Santana da Silva, S. (2019). Institutionalisation de la naissance par césarienne au Brésil et en France: composantes éducatives et sanitaires. Thèse de doctorat en sciences de l'éducation à CY Cergy Paris Université et en sciences de la santé à l'Université de Sao Paulo.

Soulière, M., Monceau, G., Fortuna, C.M., Mondain, N., Santana da Silva, S., \& Pilotti, A. (2020). Comprendre le parcours du devenir parent. Regard sur une démarche de recherche collaborative et qualitative à l'international. Enjeux et société, 7(1), 64-91.

Soulière, M., Gentelet, K. et Coman, G. (dir.). (2014). Visages contemporains de la critique sociale. Réflexions croisées sur la résistance quotidienne (p. 175-192). Canada : Editions de l'Acsalf.

\section{NOTES}

1. Etude pilote financée par l'Institut Savoir Montfort d'Ottawa, la Fondation de l'Université de Cergy-Pontoise et l'Université de São Paulo.

2. Il s'agissait presque exclusivement de femmes. Nous faisons ici le choix du féminin.

\section{RÉSUMÉS}

Cet article propose d'abord un parallèle entre le processus de réforme psychiatrique et les transformations en cours en sciences humaines et sociales, ainsi qu'un état de l'influence de la première sur les secondes. Cette entrée ancre la question de l'aliénation et l'émancipation des chercheurs dans un processus historico-politique. Les auteurs proposent ensuite une contribution concrète qui allie des visées praxéologiques, heuristiques et critiques. Ils présentent la création et la dynamique d'un réseau scientifique international (Brésil, Canada et France principalement) et interdisciplinaire. Enfin, l'analyse des activités du réseau est illustrée par un exemple de recherche qui éclaire comment les transformations des pratiques incluent la réflexivité critique des chercheurs sur leurs propres aliénations et la recherche collective de modes alternatifs de mener de activités de recherche. Il en va de l'émancipation des chercheurs, des types de connaissances produites et des personnes concernées.

This article first proposes a parallel between the process of psychiatric reform and the transformations taking place in the human and social sciences, as well as a state of the influence of the former on the latter. This entry anchors the question of alienation and emancipation of 
researchers in a historical-political process. Then, the authors propose a concrete contribution that combines praxeological, heuristic and critical aims. They present the creation and dynamics of an international (mainly Brazil, Canada and France ) and interdisciplinary scientific network. Finally, the analysis of the network's activities illustrated by a research example sheds light on how the transformations of practices include the critical reflexivity of researchers on their own alienations and the collective search for alternative modes of conducting research activities. At stake is the emancipation of researchers, the types of knowledge produced and the people involved.

INDEX

Mots-clés : aliénation en recherche, pratiques de recherche émancipatoires, réflexivité critique, réseau

Keywords : alienation in research; emancipatory research practices; critical reflexivity; research network with

\section{AUTEURS}

\section{GILLES MONCEAU}

EMA, CY Cergy Paris Université

\section{MARGUERITE SOULIÈRE}

Faculty of Social Sciences, School of Social Work

\section{CINIRA MAGALI FORTUNA}

NUPESCO, Escola de Enfermagem de Ribeirão Preto da Universidade de São Paulo 